\title{
Correlation of mixed lymphocyte culture with chronic graft-versus-host disease following allogeneic stem cell transplantation
}

J.E.L. Visentainer,

S.R. Lieber,

L.B.L. Persoli,

S.C.B. de Souza Lima,

A.C. Vigorito, F.J.P. Aranha,

K.A.B. Eid, G.B. Oliveira,

E.C.M. Miranda and

C.A. de Souza
Faculdade de Ciências M édicas, Universidade Estadual de Campinas, Campinas, SP, Brasil

\section{Correspondence}

J.E.L. Visentainer

R. Arthur Thomas, 259

Apto. 2101

87013-297 Maringá, PR

Brasil

Fax: + 55-19-3788-8600

E-mail: jelvisentainer@ ibest.com.br

and jelvisentainer@uem.br

Publication supported by FAPESP.

Received November 5, 2001

Accepted March 14, 2002

\section{Abstract}

The purpose of the present study was to evaluate the mixed lymphocyte culture as a predictive assay of acute and chronic graft-versushost disease (GVHD). We studied 153 patients who received a first bone marrow transplantation from human leukocyte antigen-identical siblings. Acute GVHD was observed in 26 of 128 (20.3\%) patients evaluated and chronic GVHD occurred in 60 of 114 (52.6\%). Oneway mixed lymphocyte culture (MLC) assays were performed by the standard method. MLC results are reported as the relative response (RR) from donor against patient cells. The responses ranged from -47.0 to $40.7 \%$, with a median of $0.5 \%$. The Kaplan-Meier probability of developing GVHD was determined for patients with positive and negative MLC. There was no significant difference in incidence of acute GVHD between the groups studied. However, the incidence of chronic GVHD was higher in recipients with RR $>4.5 \%$ than in those with $\mathrm{RR} \leq 4.5 \%$. The Cox Proportional Hazards model was used to examine the effect of MLC levels on incidence of chronic GVHD, while adjusting for the potential confounding effect of others suspected or observed risk factors. The relative risk of chronic GVHD was 2.5 for patients with positive MLC (RR $>4.5 \%$ ), 2.9 for those who received peripheral blood progenitor cells as a graft, and 2.2 for patients who developed previous acute GVHD. MLC was not useful for predicting acute GVHD, but MLC with RR $>4.5 \%$ associated with other risk factors could predict the development of chronic GVHD, being of help for the prevention and/or treatment of this late complication.

\section{Key words}

- Mixed lymphocyte culture

- Allogeneic stem cell transplantation

- Chronic graft-versus-host disease 


\section{Introduction}

The development of acute and chronic graft-versus-host disease (GVHD) is still a severe complication in allogeneic bone marrow transplantation (BMT) and depends on multiple factors (1). The selection of bone marrow donors has been based on serology and on DNA-based techniques for definition of human leukocyte antigen (HLA) compatibility. Mixed lymphocyte culture (MLC) has been proposed as a functional test to measure in vitro allo-responsiveness and has been used to select matched recipients and donors in BMT for over 30 years (2). The development and routine use of high resolution DNA typing techniques for MHC II alleles has led to different opinions on the importance of MLC for the selection of BMT donors (3). Although the MLC had become somewhat redundant as a prospective assay of compatibility, it has permitted the evaluation of the stimulatory capacity of different class II gene products and may contribute to the decision on the suitability of a potential donor. In the present study, we analyzed the correlation between MLC results and GVHD in BMT involving HLA-identical siblings.

\section{Patients and Methods}

\section{Patients and donors}

A total of 153 patients aged 3 to 59 years (median: 29 years) with hematological malignancies, who underwent a first allogeneic BMT at the Hematology and Hemotherapy Center, State University of Campinas, from September 1993 to November 1999, were followed up for clinical outcome up to July 31,2001 . The present study included only patients who received an allogeneic BMT from HLA-identical siblings with available MLC data. One hundred and thirteen patients received bone marrow as a graft and 40 patients received peripheral blood pro- genitor cells (PBPC). In the evaluation of acute GVHD, 25 patients were excluded from the analysis because 18 had died without acute GVHD and 7 had received marrow reinfusion before day 100 , and in the evaluation of chronic GVHD, 14 additional patients were excluded because they died before day 100 with acute GVHD, leaving a total of 114. The patient and treatment characteristics are listed in Table 1. The present study was performed according to the Ethics Committee of the Hematology and Hemotherapy Center.

\section{HLA typing}

Patients and donors were HLA-A, -B, -DR and -DQ typed using monoclonal antibodies (One Lambda ${ }^{\circledR}$, Canoga Park, CA, USA) or alloimmune sera (Pel-Freez ${ }^{\mathbb{R}}$, Brow Deer, WI, USA) up to October 1997. Low resolution molecular typing of DRB1 and DQB1 was then performed using the Dynal ${ }^{\circledR}$ sequence-specific primer (Dynal Ltd., Bromborough, Wirral, UK).

\section{Mixed lymphocyte culture}

One-way MLC assays were performed according to standard techniques. Briefly, mononuclear cells were isolated from heparinized blood by flotation over Ficoll and resuspended in culture medium. Mononuclear responder cells $\left(1 \times 10^{5}\right)$ were cultured in triplicate in trays with $1 \times 10^{5}$ mitomycin-C-treated stimulator cells for 6 days in a humidified atmosphere with $5 \% \mathrm{CO}_{2}$. Cultures were pulse labeled with tritiated thymidine and further incubated for $20 \mathrm{~h}$ at $37^{\circ} \mathrm{C}$. After harvesting by aspiration onto glass fiber filters, total isotope incorporation was determined with a scintillation counter per minute. The MLC levels were expressed as mean counts per minute in triplicate cultures and were standardized for purposes of comparison according to procedures previously described (4): 


$$
\mathrm{RR}(\%)=\frac{\text { Allogeneic MLC - autologous MLC }}{\text { Maximum MLC - autologous MLC }} \times 100 .
$$

The reference response value is equated to the maximum response obtained for the particular responder cell in the experiment; this is usually provided by one of the individual unrelated control cells or by the pool of unrelated control cells. The ratio is usually multiplied by 100 to yield a "percent relative response (RR) value".

\section{Transplantation procedures}

All patients were prepared for BMT according to protocols appropriate for their underlying and overt disease. Bone marrow was obtained from donors by standard methods. All PBPC donors received rhGCSF (Granulokine; Roche, São Paulo, SP, Brazil) by subcutaneous injection as described elsewhere (5). Day 0 for the recipients was defined as the day of bone marrow or PBPC infusion. A summary of pretreatment preparative regimens and GVHD prophylaxis is shown in Table 1. PBPC and bone marrow were analyzed for CD34+ cells and T-cell subsets by flow cytometry. Monoclonal antibodies were obtained from Becton Dickinson ${ }^{\circledR}$ Immunocytometry Systems (San Jose, CA, USA). Neutrophil engraftment was considered to have occurred on the first of two consecutive days with an absolute neutrophil count $>0.5 \times 10^{9} / 1$. Platelet engraftment was considered to have occurred on the first of seven consecutive days with a platelet count $>20.0 \times 10^{9} / 1$ without platelet transfusion. Engraftment was also documented by marrow aspiration, biopsy and cytogenetic assessment and grading of acute and chronic GVHD was performed using the Glucksberg (6) and Shulman (7) criteria, respectively. In this study, only disease of grade two or more was considered to be acute GVHD.

\section{Clinical support}

Supportive care was provided in private rooms equipped with HEPA filters. Irradiated red blood cell and platelet transfusions, as well as broad-spectrum antibiotics together with anti-fungal agents, were given whenever indicated. Ceftazidime administration was started as soon as the neutrophil count dropped below $0.5 \times 10^{9} / 1$ and changes in antibiotics were made as indicated by

Table 1. Patient and treatment characteristics.

\begin{tabular}{ll}
\hline Number of patients & 153 \\
Patient age (median) & $3-59$ years (29) \\
Donor/patient gender & $\mathrm{M} / \mathrm{M}=51 / 97(52.6 \%)$ \\
& $\mathrm{F} / \mathrm{M}=46 / 97(47.4 \%)$ \\
& $\mathrm{M} / \mathrm{F}=26 / 56(46.4 \%)$ \\
& $\mathrm{F} / \mathrm{F}=30 / 56(53.6 \%)$ \\
Conditioning regimen & $\mathrm{Bu}+\mathrm{Cy}=44(28.7 \%)$ \\
& $\mathrm{Bu}+\mathrm{Cy} 2=82(53.6 \%)$ \\
& $\mathrm{Bu}+\mathrm{Cy}+\mathrm{VP}=9(5.9 \%)$ \\
& $\mathrm{Cy}=6(3.9 \%)$ \\
& $\mathrm{Cy}+\mathrm{ATG}=1(0.7 \%)$ \\
First BMT graft & $\mathrm{Cy2}+\mathrm{TBI}=10(6.5 \%)$ \\
BM & $\mathrm{Cy}+\mathrm{Mel}=1(0.7 \%)$ \\
PBPC & \\
GVHD prophylaxis & $113(73.9 \%)$ \\
& $40(26.1 \%)$ \\
& $\mathrm{MTX}+\mathrm{CsA}=141(92.2 \%)$ \\
Malignant diseases & $\mathrm{CsA}=2(1.3 \%)$ \\
Early disease (CML, 1st CP; AML, 1st CR; & $\mathrm{CsA}+\mathrm{Cort}=10(6.5 \%)$ \\
AML, 1st rel; ALL, 1st CR) & \\
Late disease (CML, AP/BC; AML $>1$ 1st rel; & $91(59.5 \%)$ \\
refractory AML, ALL $>2$ nd) & $33(21.6 \%)$ \\
Other malignant and non-malignant diseases & \\
\hline
\end{tabular}

$M$, male; $F$, female; BMT, bone marrow transplantation; BM, bone marrow; PBPC, peripheral blood progenitor cells; GVHD, graft-versus-host disease; $C M L$, chronic myeloid leukemia; $C P$, chronic phase; $A M L$, acute myeloid leukemia; $C R$, complete remission; rel, relapse; $A L L$, acute lymphoblastic leukemia; AP/BC, accelerated phase/ blastic crisis; AA, aplastic anemia; PNH, paroxysmal nocturnal hemoglobinuria; MDS, myelodysplastic syndrome; NHL, non-Hodgkin's lymphoma; MM, multiple myeloma; DBA, Diamond-Blackfan anemia; CMML, chronic myelomonocytic leukemia; DAC, dyserythropoietic anemia congenital; Bu, busulfan; Cy, cyclophosphamide; VP, etoposide; ATG, antithymocyte globulin; TBI, total body irradiation; Mel, melphalan; MTX, methotrexate; CsA, cyclosporine; cort, corticosteroid. 
Figure 1. Incidence of chronic graft-versus-host disease (GVHD) following bone marrow transplantation as a function of the relative response (RR) of mixed lymphocyte culture.
Figure 2. Incidence of chronic graft-versus-host disease (GVHD) following bone marrow transplantation as a function of graft source. PBPC, peripheral blood progenitor cells; BM, bone marrow. cultures and clinical course, keeping the patient on antibiotics until the granulocyte count reached $1.0 \times 10^{9} / 1$. All patients received Ganciclovir ( $5 \mathrm{mg} \mathrm{kg}^{-1}$ day-1 $^{-1}$ three times a week) after bone marrow recovery as a prophylactic measure for cytomegalovirus infection until day 75 post-transplant. Nutritional support, including total intravenous nutrition, was provided to assure an adequate nutritional balance.

\section{Statistical analysis}

Patients were classified as having no acute or chronic GVHD at the time of marrow reinfusion or at the time of death. The KaplanMeier methods and the log-rank test were used to determine the probability of acute and/or chronic GVHD. Regarding MLC, the cut-off points were found based on the receiver operator characteristic curve and on literature (8). A cut-off point of $4.5 \%$ was established for chronic GVHD and a cut-off point of 5\% for acute GVHD.
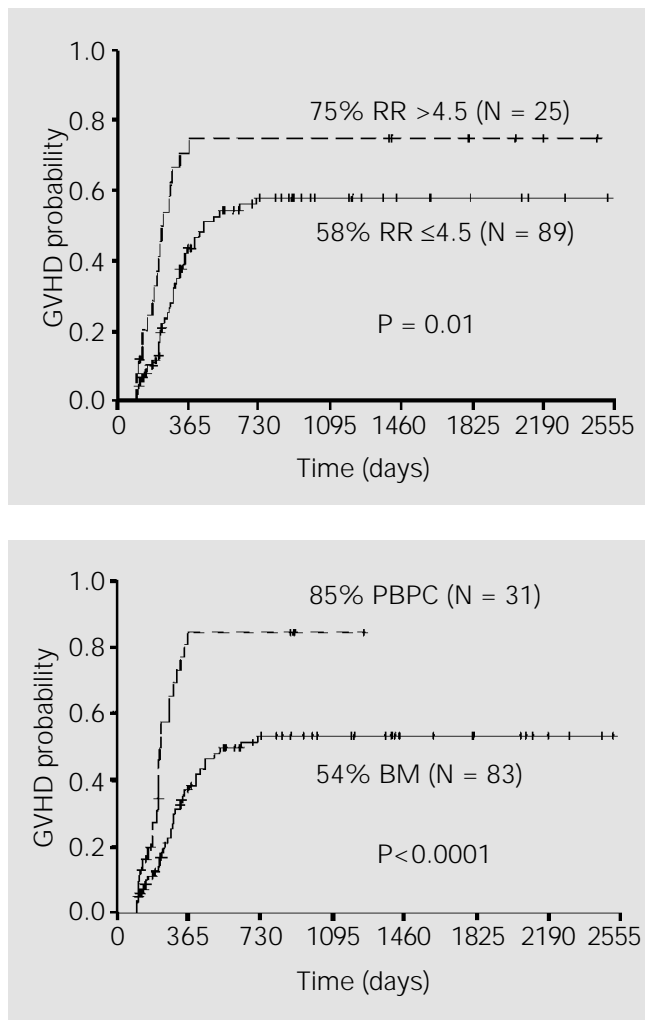

The variables included were patient age, sex, disease status, graft source (bone marrow or PBPC), prior acute GVHD and positive MLC, which were examined for association with chronic GVHD. Factors associated with the risk of chronic GVHD in univariate analysis at the $\mathrm{P}<0.20$ level of significance were examined by multivariate analysis using proportional hazards regression (Cox Model). All P values $\leq 0.05$ were considered to be significant. The SAS System version 6.12 for Windows 95 and SPSS software version 10.0.5 were used.

\section{Results}

\section{Relative response values and clinical outcome}

The RR values ranged from -47.0 to $40.7 \%$ (median, 0.5 ) in 153 donor versus recipient responses analyzed by the MLC method. Acute GVHD of grade two or higher was observed in $20.3 \%(26 / 128)$ of patients, distributed as follows: $23.1 \%$ grade $2,30.8 \%$ grade 3 and $46.2 \%$ grade 4 . Chronic GVHD was observed in $52.6 \%(60 / 114)$ of patients, limited in $23.3 \%$ and extensive in $76.7 \%$. Thirteen percent of cases had prior acute GVHD.

\section{Risk of acute and chronic GVHD}

Using standard life-table methods and regression models, no association was found between acute GVHD and any of the variables tested. Kaplan Meier estimates showed that chronic GVHD was observed in $75 \%$ of recipients with $\mathrm{RR}>4.5 \%$ and in $58 \%$ of those with $R R \leq 4.5 \%(P=0.01)$ (Figure 1). The probability of chronic GVHD was increased in the group that received PBPC compared to the group that received bone marrow (85 vs 54\%, $\mathrm{P}<0.0001$ ) (Figure 2) and the group that had developed prior acute GVHD (78 vs 60\%, P = 0.04) (Figure 3).

Using the Cox Proportional Hazards mo- 
del, the risk of earlier chronic GVHD was observed when RR was $>4.5 \%$, when patients had received PBPC as a graft or when the recipients had already developed acute GVHD (Table 2). When continuous RR was used, the relative risk of earlier chronic GVHD increased by 1.0 for each RR unit (P $=0.004)$; for PBPC as graft cells the risk was $2.5(\mathrm{P}=0.001)$, and for previous acute GVHD the risk was $2.2(\mathrm{P}=0.03)$. The median time to onset of chronic GVHD for patients with RR $>4.5 \%$ was 201 days and for those with $\mathrm{RR} \leq 4.5 \%, 233$ days. For patients who received bone marrow as graft this median time was 238.5 days and for those who received PBPC it was 214.5 days. The median time was 147.0 days for patients with previous acute GVHD factor and 228.5 days for those without this factor.

\section{Discussion}

We observed acute GVHD in $20.3 \%$ of HLA-matched siblings and chronic GVHD in $52.6 \%$. Disparities in minor histocompatibility antigen HA-1 (9) and HLA-DP incompatibilities (10) could be involved in the development of acute GVHD. Another possibility is that post-transplant immunological tolerance, including suppressive mechanisms, may influence the development of GVHD (11). The use of the MLC assay has been questioned as a predictor of acute GVHD in HLA-identical sibling transplants $(12,13)$. The authors suggested that conventional MLC could be omitted in HLA-A, -B and -DR compatible sibling bone marrow transplants. Our data did not demonstrate any association between acute GVHD and MLC. It is possible that other factors not related to HLA or specific DP disparity not detectable in the MLC can lead to acute GVHD.

Nevertheless, MLC results seemed to be important for predicting chronic GVHD because GVHD occurred more frequently and earlier in recipients who showed positive
MLC (RR >4.5\%). The development of chronic GVHD is complex and it is not completely understood. At least two phenomena can be associated with it, the decrease of graft monocytes and the maintenance of T-cells for a long time after BMT. These types of cells are producers of cytokines that can act as defenders or promote inflammation (14). The MLC reflects the capacity of T-cells to produce the cytokines through an allogeneic stimulus.

Evidence favoring distinct mechanisms of acute and chronic GVHD includes the difference in clinical manifestations. Both of them may affect multiple solid organs, but the chronic form has been correlated with the development of autoimmune-like sequels due to autoreactive T lymphocytes (15). Thus, development of different T-cell subsets may influence the clinical manifestations of the alloreactive component of GVHD.

Furthermore, we confirmed previous reports from our group and others that have shown a high incidence and/or severity of chronic GVHD after PBPC grafting $(5,14)$ and after acute GVHD (16).

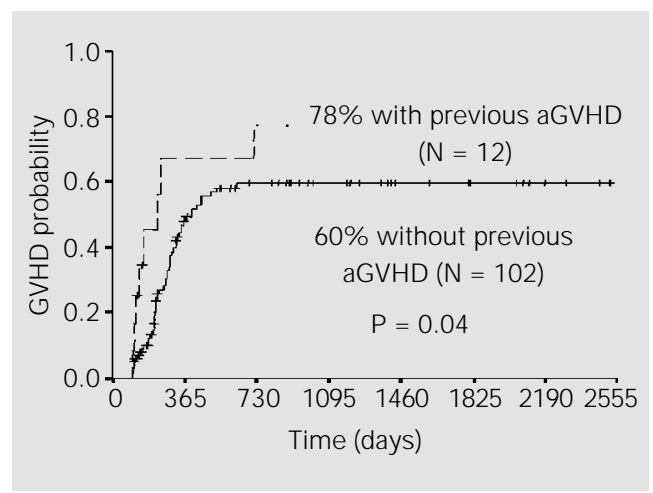

Figure 3. Incidence of chronic graft-versus-host disease (GVHD) following bone marrow transplantation according to previous acute GVHD (aGVHD).
Table 2. Factors involving chronic graft-versus-host disease (GVHD) risk.

\begin{tabular}{lcccl}
\hline Statistical method & Variables & Relative risk & $\mathrm{Cl}(95 \%)$ & P value \\
\hline Multivariate Cox & MLC (RR $>4.5 \%)$ & 2.5 & $1.4-4.5$ & 0.001 \\
& PBPC graft & 2.9 & $1.6-5.1$ & 0.0002 \\
Proportional Hazards & Previous acute GVHD & 2.2 & $1.0-4.9$ & 0.03 \\
\hline
\end{tabular}

$\mathrm{Cl}$, confidence interval; MLC, mixed lymphocyte culture; RR, donor versus recipient relative response; PBPC, peripheral blood progenitor cells. 
Although today DNA-based techniques have improved HLA typing, our data suggest that MLC reactivity may be clinically useful in predicting the development of chronic
GVHD. MLC would enable physicians to predict the need for additional treatment in order to minimize the allogeneic BMT complications.

\section{References}

1. Ferrara J LM (2000). Pathogenesis of acute graft-versus-host disease: cytokines and cellular effectors. J ournal of Hematotherapy and Stem Cell Research, 9: 299306.

2. Bach FH \& Hirschhorn K (1964). Lymphocyte interaction: a potential histocompatibility test in vitro. Science, 143: 813814.

3. Pénzes M, Rajczy K, Nyári E, Gyódi É \& Petrányi Gy (1996). The importance of mixed lymphocyte culture (MLC) in the donor selection for bone marrow transplantation. Human Immunology, 47: 85 (Abstract).

4. Mickelson EM, Guthrie LA \& Hansen J A (2000). The mixed lymphocyte culture (MLC) test. In: Land G \& Strothman R (Editors), ASHI Laboratory Manual. The American Society for Histocompatibility and Immunogenetics, New York, NY, USA.

5. Vigorito AC, Azevedo WM, Marques J FC, Azevedo AM, Eid KAB, Aranha FJP, Lorand-M etze I, Oliveira GB, Correa MEP, Reis ARC, Miranda ECM \& de Souza CA (1998). A randomized, prospective comparison of allogeneic bone marrow and peripheral blood progenitor cell transplantation in the treatment of hematological malignancies. Bone Marrow Transplantation, 22: 1145-1151.

6. Glucksberg H, Storb R \& Fefer A (1974). Clinical manifestation of graft-versus-host disease in human recipients of marrow from HLA matched sibling donors. Transplantation, 18: 295-314.
7. Shulman HM, Sullivan $K M \&$ Weiden $P L$ (1980). Chronic graft-versus-host syndrome in man. A long-term clinicopathologic study of 20 Seattle patients. American J ournal of Medicine, 69: 204-217.

8. Al-Daccak R, Loiseau P, Soulie A, Varrin F, Rabian C, Raffoux C, Cohen D, Degos L, Gluckman E \& Colombani J (1990). HLADP genotyping in HLA-A, B, and DR identical interfamilial bone marrow transplantation. Leukemia, 4: 222-226.

9. Goulmy E, Shipper R, Pool J, Blokland E, Falkenburg J HF, Vossen J, Gratwohl A, Vogelsang GB, van Houwelinges HC \& van Rood J J (1996). Mismatches of minor histocompatibility antigen between HLAidentical donors and recipients and the development of graft-versus-host disease after interfamilial bone marrow transplantation. New England J ournal of Medicine, 334: 281-285.

10. Nomura N, Ota M, Kato S, Inoko H \& Tsuji K (1991). Severe acute graft-versus-host disease by HLA-DPB1 disparity in recombinant family of bone marrow transplantation between serologically HLA-identical siblings: an application of the polymerase chain reaction - restriction fragment length polymorphism method. Human Immunology, 32: 261-268.

11. Tsoi MS, Storb R \& Dobbs S (1981). Specific suppressor cells in graft-host tolerance of HLA-identical marrow transplantation. Nature, 292: 355-357.

12. Lim SH, Patton WN, J obson S, Gentle TA, Baynham MID, Franklin IM \& Boughton BJ (1988). Mixed lymphocyte reactions do not predict severity of graft versus host disease (GVHD) in HLA-DR compatible, sibling bone marrow transplants. J ournal of Clinical Pathology, 41: 11551157.

13. van der Meer A, Allebes WA, Voorter CEM, van den Berg-Loonen EM, Schattenberg AV, de Witte TJ \& J oosten I (1998). Helper and cytotoxic T cell precursor frequencies are not predictive for development of acute graft-versus-host disease after partially $\mathrm{T}$ cell-depleted HLAidentical sibling BMT. Bone Marrow Transplantation, 22: 1049-1055.

14. Storek J, Gooley T, Siadak M, Bensinger WI, Maloney DG, Chauncey TR, Flowers $M$, Sullivan KM, Witherspoon RP, Rowley SD, Hansen J A, Storb R \& Appelbaum FR (1997). Allogeneic peripheral blood stem cell transplantation may be associated with a high risk of chronic graft-versushost disease. Blood, 90: 4705-4709.

15. Murphy WJ (2000). Revisiting graft-versus-host disease models of autoimmunity: new insights in immune regulatory process. J ournal of Clinical Investigation, 106: 745-747.

16. Atkinson K, Horowitz MM, Gale RP, van Bekkum DW, Gluckman E, Good RA, J acobsen N, Kolb HJ , Rimm AA, Ringdén O, Rozman C, Sobocinski KA, Zwaan FE \& Bortin MM (1990). Risk factors for chronic graft-versus-host disease after HLA-identical sibling bone marrow transplantation. Blood, 75: 2459-2464. 